\title{
Synchronization in networks of mobile oscillators
}

\author{
Naoya Fujiwara \\ Potsdam Institute for Climate Impact Research (PIK), 14473 Potsdam, Germany
}

Jürgen Kurths

Potsdam Institute for Climate Impact Research (PIK), 14473 Potsdam, Germany and Institute for Complex Systems and Mathematical Biology, University of Aberdeen, UK

\author{
Albert Díaz-Guilera \\ Departament de Física Fonamental, Universitat de Barcelona, 08028 Barcelona, Spain and \\ Potsdam Institute for Climate Impact Research (PIK), 14473 Potsdam, Germany
}

(Received 13 December 2010; published 7 February 2011)

\begin{abstract}
We present a model of synchronization in networks of autonomous agents where the topology changes due to agents motion. We introduce two timescales, one for the topological change and another one for local synchronization. If the former scale is much shorter, an approximation that averages out the effect of motion is available. Here we show, however, that the time required for synchronization achievement is larger than the prediction of the approximation in the opposite case, especially close to the continuum percolation transition point. The simulation results are confirmed by means of spectral analysis of the time-dependent Laplacian matrix. Our results show that the tradeoff between these two timescales, which have opposite effects on synchronization, should be taken into account for the design of mobile device networks.
\end{abstract}

DOI: 10.1103/PhysRevE.83.025101

PACS number(s): $89.75 . \mathrm{Hc}, 05.45 . \mathrm{Xt}$

After an initial period of characterizing complex networks in terms of local and global statistical properties (e.g., Ref. [1]), attention turned to the dynamics of their interacting units [2]. A widely studied example of such behavior is synchronization of coupled oscillators arranged into complex networks. The interplay between topology and dynamics is crucial for synchronization achievement (see Ref. [3] and references therein). In most studies of such systems the network has a fixed structure, but there are also many interesting scenarios where the topology changes over time in various fields, such as power transmission system [4], consensus problem [5], mobile communication [6], and functional brain networks [7].

Within the general framework of time-dependent or evolving networks, we can identify the particular case of networks whose nodes represent physical agents that move around but interact with each other only when they are close enough. Examples include the coordinated motions of robots [8], vehicles [9], and groups of animals [10], in which cooperative dynamics emerge. Especially, there are many examples where synchronization plays a crucial role: chemotaxis [11], mobile ad hoc networks [12], and wireless sensor networks [13]. Despite the importance of this topic, prior research on synchronization in time-dependent networks of populations of agents has concentrated so far on two special cases: (i) where the network topology changes fast [14-17] and (ii) where the population is dense and arranged in a ring [18]. In the former case, the fast-switching approximation (FSA), which averages out the effect of agent motion, is commonly used. However, for better understanding of synchronization of mobile agents and design of an efficient network, it is very important to clarify when and how FSA fails.

In order to study this point, this Rapid Communication proposes a general framework in which agents perform random walks in a two-dimensional (2D) plane. We consider that each agent possesses a mobile wireless device whose state is characterized by a phase variable, and the phases approach one another through the interaction between agents within a certain spatial range. This model is well suited for communication problems with short-range wireless devices. In this Rapid Communication we show a general mechanism of failure of FSA when the timescale of local synchronization is shorter than the timescale of the topology change due to the agent motion. Since we need longer synchronization time due to this failure, it is an important factor we should take into account for constructing an efficient mobile network.

Our model consists of $N$ agents moving in a 2D space (size $L \times L$ ) with periodic boundary conditions. Each agent moves with velocity $v$. The angle of the $i$ th agent's motion is $\xi_{i}\left(t_{k}\right) \in[0,2 \pi]$, and it changes randomly at discrete time steps $t_{k}\left(t_{k+1}-t_{k}=\tau_{M}\right)$. The evolution of the $i$ th agent's position is therefore

$$
\begin{aligned}
& x_{i}\left(t_{k}+\Delta t\right)=x_{i}\left(t_{k}\right)+v \cos \xi_{i}\left(t_{k}\right) \Delta t \quad \bmod L, \\
& y_{i}\left(t_{k}+\Delta t\right)=y_{i}\left(t_{k}\right)+v \sin \xi_{i}\left(t_{k}\right) \Delta t \bmod L,
\end{aligned}
$$

where $\Delta t \leqslant \tau_{M}$. The motion of the agents is diffusive, with a diffusion coefficient of $D \sim v^{2} \tau_{M}$.

In this Rapid Communication the dynamics of the oscillators are based on the Kuramoto model [19], which has been applied to technological problems recently [20]. The time evolution of the phase of oscillator $i$ is represented as

$$
\varphi_{i}\left(t+\tau_{P}\right)=\varphi_{i}(t)+\sigma \sum_{j, d_{i j}<d} \sin \left[\varphi_{j}(t)-\varphi_{i}(t)\right],
$$

where $d_{i j}=\sqrt{\left(x_{i}-x_{j}\right)^{2}+\left(y_{i}-y_{j}\right)^{2}}$. Since mobile devices emit signals at discrete time intervals, the individual phases are updated at discrete time steps of duration $\tau_{P}$. Only devices within a distance $d$ of each other can interact to approach their phases. 
When the phase difference is small, Eq. (2) can be well approximated by the linearized equation

$$
\varphi_{i}\left(t+\tau_{P}\right)=\varphi_{i}(t)-\sigma \sum_{j=1}^{N} L_{i j}(t) \varphi_{j}(t),
$$

which provides interesting hints about the dynamical behavior. $L_{i j}(t)=\left[k_{i}(t) \delta_{i j}-c_{i j}(t)\right]$ is the time-dependent Laplacian matrix with $c_{i j}(t)=1$ if $d_{i j}<d \quad(i \neq j)$ and $c_{i j}(t)=0$ otherwise. $k_{i}(t)$ represents the number of oscillators that are around $i$ within a range $d$.

We first note that the instantaneous coordination of agents has statistical properties similar to those of a continuum percolation. Simply changing $d$ can be enough for the system to enter a different topological (static) configuration. The transition takes place at $(N-1) \pi d_{c}^{2} / L^{2} \approx 4.51$ (see Ref. [21] and references therein). In this Rapid Communication we fix $N=100, L=200, v=10$, and $\tau_{M}=1$, which imply $d_{c} \approx 24.1$. Since agents move and the system is finite, we do not observe a sudden transition at $d=d_{c}$.

Starting from random initial phases and positions, we let the system evolve according to Eqs. (1) and (2). Figure 1 presents time sequences of four snapshots for three different sets of parameters [22]. Synchronization emerges through motion and intermittent communication between agents, even though a single connected component never forms below $d_{c}$ [Figs. 1(a) and 1(b)]. Above $d_{c}$ [Fig. 1(c)], agent motion is not necessary but still helps the system to reach its final state.

In all our simulations, the average phase difference $\langle\Delta \varphi\rangle \equiv$ $\sqrt{\frac{2}{N(N-1)} \sum_{j<k}\left(\varphi_{j}-\varphi_{k}\right)^{2}}$ decays exponentially after an initial transient. We can define then a characteristic time $T$ in such a way that $\langle\Delta \varphi\rangle \propto e^{-t / T}$ and estimate $T$ by fitting the numerical data of $\langle\Delta \varphi\rangle$. Additionally, $n_{T} \equiv T / \tau_{P}$ stands for the number of phase updates the system needs to reach complete synchronization, and its inverse defines the system efficiency. Minimizing $n_{T}$ leads to more efficient use of the mobile devices' batteries.
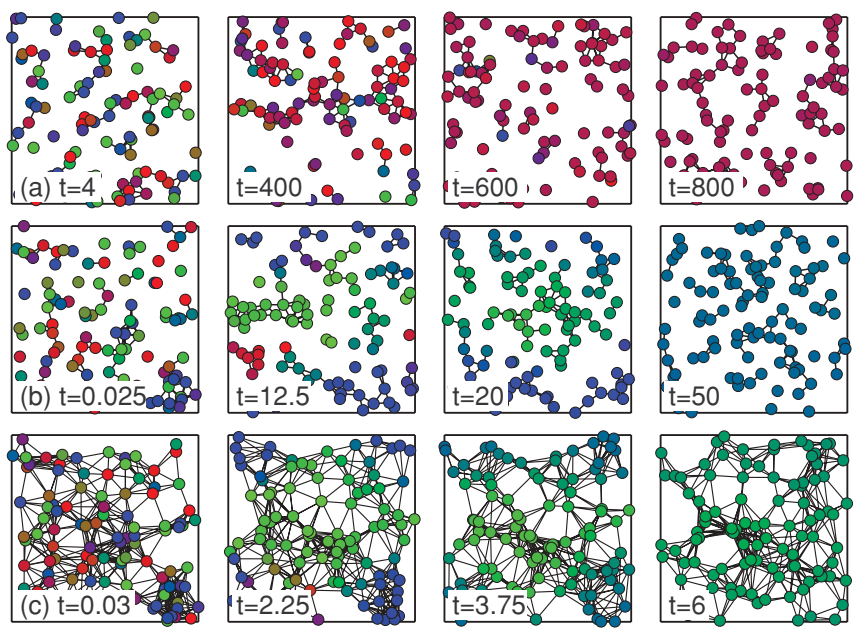

FIG. 1. (Color online) Time evolution of the positions and phases of the agents. The colors are mapped to phases on the interval $[0,2 \pi]$; lines are drawn between two nodes when their separation is less than $d$. Each row corresponds to a different set of parameters: $\tau_{P}=1$, $d=20<d_{c}$ for (a), $\tau_{P}=0.01, d=20$ for (b), and $\tau_{P}=0.01, d=$ $40>d_{c}$ for $(\mathrm{c})$.
In the literature related to interacting units in timedependent networks, authors often use the FSA [14-17]. FSA assumes that the topology changes fast enough, and the entries in the connectivity matrix are replaced by the probability that two units are within the interaction range under completely random motion $\left(\rho=\pi d^{2} / L^{2}\right.$, for $\left.d \leqslant L / 2\right)$. The characteristic time $T_{\mathrm{FS}}$ within FSA is expressed as

$$
T_{\mathrm{FS}}=-\tau_{P} / \log [1-\sigma(N-1) \rho] .
$$

The effect of agents motion is averaged out, and the parameters $v$ and $\tau_{M}$ do not appear in Eq. (4). It is important to note that it makes sense only when the timescale of network variations is much shorter than that of the interaction.

Figure 2(a) plots $n_{T}$ as a function of $d$ for various values of $\tau_{P}$. As intuitively expected, there is a decreasing monotonic relationship between $n_{T}$ and $d$. We have also drawn a reference line along $T_{\mathrm{FS}} / \tau_{P}$, which depends only on $d$. For large $\tau_{P}$ FSA is very accurate over a wide range of $d$, since the mentioned condition for FSA is satisfied. However, we also identify a region in Fig. 2(a) where FSA does not hold for intermediate values of $d$. The size of this region increases as $\tau_{P}$ decreases.

It is important to note that these deviations from FSA take place close to the continuum percolation transition point. This is not a sharp transition; instead, $T$ gradually deviates from FSA. To gain a broader understanding, we plot $T / T_{\mathrm{FS}}$ over the $\tau_{P}-d$ plane in Fig. 2(b). For large enough $\tau_{P}$, FSA is very good irrespective of $d$.

In order to qualitatively explain the deviations from FSA, we consider two characteristic timescales: one for clusters to synchronize and another one for breaking apart. The number of time steps (measured in units of $\tau_{P}$ ) for a cluster to synchronize is, to first order in $\sigma, n_{s}=1 / \sigma \lambda_{2}^{c}(d)$. Here $\lambda_{2}^{c}$ stands for the smallest nonzero eigenvalue of the Laplacian of the cluster. On the other hand, the number of steps for a single oscillator to leave a cluster of size $\xi(d)$ (an increasing function of $d$ if $d<$ $d_{c}$ ) is $n_{m}=\xi^{2}(d) / v^{2} \tau_{M} \tau_{P}$. We can then introduce the ratio

$$
\eta=\frac{n_{m}}{n_{s}}=\frac{\sigma f(d)}{v^{2} \tau_{M} \tau_{P}}
$$

which gives us the dominant timescale, where $f(d) \equiv$ $\xi^{2}(d) \lambda_{2}^{c}(d)$. Note that the topological parameter $d$ (also $N$ and $L$, if they were changed) appears only in $f$, while the parameters related to agent dynamics appear only in the denominator.

It is clear from Eq. (5) that $\eta$ decreases if we increase $\tau_{P}$. This fact predicts a transition in the dominant timescale as we change $\tau_{P}$, which is confirmed in Fig. 2. We expect the same transition in $f(d)$ by changing $d$. Our numerical results in Fig. 2 suggest that $f(d)$ is an increasing function of $d$ well below $d_{c}$. In the following, we analyze three different asymptotic behaviors in detail:

(i) The condition $\eta \ll 1$ holds for small $d$ and large $\tau_{P}$ in Fig. 2. In this region, the displacement of agents between $\tau_{P}$ is large. Thus, the network connectivity changes very fast before agents synchronize with their neighbors. Figure 1(a) shows the evolution of a system under these conditions. All nodes in the system approach complete synchronization at approximately the same rate, and nonsynchronized nodes may become spatially isolated. We call this mechanism global synchronization. FSA is accurate for systems of this type. 

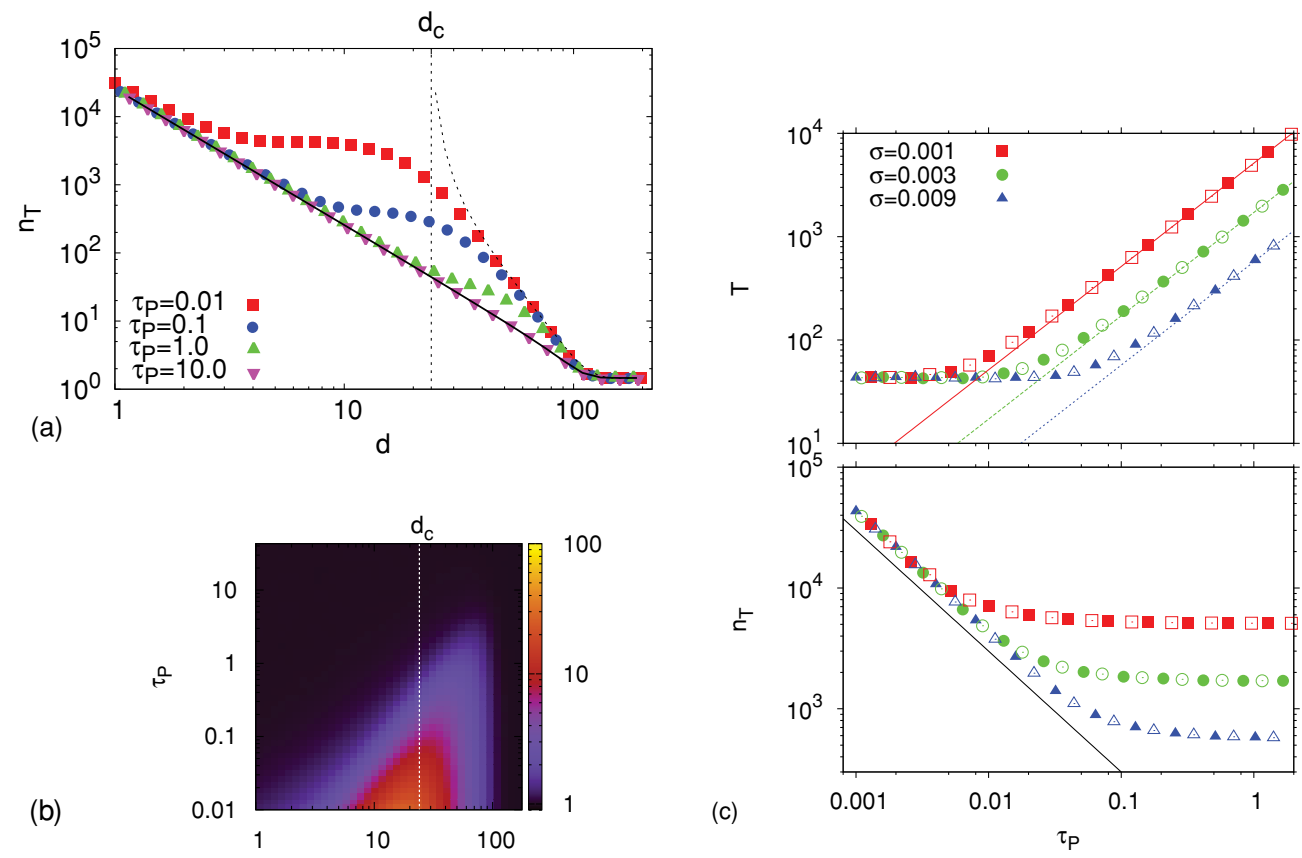

FIG. 2. (Color online) (a) Number of updates required to synchronize $n_{T}=T / \tau_{P}$ as a function of $d$. The solid line indicates FSA, $T_{\mathrm{FS}} / \tau_{P}$ [Eq. (4)]. The dotted line is $T_{\mathrm{SL}} / \tau_{P}$ [Eq. (6)]. (b) The ratio $T / T_{\mathrm{FS}}$ in the $\tau_{P}-d$ plane. In (a) and (b) $\sigma=0.005$, and $d_{c}$ represents the continuum percolation threshold for an infinite system [21]. (c) $T$ and $n_{T}$ versus $\tau_{P}$ with different values of $\sigma$ for $d=5$. Filled and open symbols of the same color are based on Eq. (2) and the matrix product formulation (8), respectively. The colored lines in the top panel represent $T_{\mathrm{FS}}$ for each case. The black line in the bottom panel represents $\tau_{P}^{-1}$.

(ii) Starting from the previous case, $\eta$ increases when either $d$ is increased or $\tau_{P}$ is decreased. For $d \ll d_{c}, \eta>1$ implies that the number of time steps required for agent motions to rewire disconnected clusters is larger than that required for synchronization inside an isolated cluster. Local clusters therefore synchronize very easily before the topology changes [Fig. 1(b)]. The dynamics toward complete synchronization is limited by the motion of the agents, and we call this mechanism multiple cluster local synchronization. Consecutive positions of agents are correlated, and the evolution of the system depends heavily on the details of the connectivity pattern. Since FSA neglects such a correlation, it does not properly describe the synchronization dynamics in this case. Indeed, as shown in Fig. 2(b), FSA fails by orders of magnitude. It is clear from Eq. (5) that this region is broader if $\tau_{P}$ is smaller.

(iii) For $d \gg d_{c}$ and $\eta>1$ (implying small $\tau_{P}$ ), the whole network is connected (single cluster). In this case, agent motion is not necessary for the final synchronization. For a static connected network the characteristic time is $T_{\text {static }}=-\tau_{P} / \log \left(1-\sigma \lambda_{2}\right)$, where $\lambda_{2}$ is the second-smallest eigenvalue of the Laplacian matrix [3]. When the network topology changes, its time average

$$
T_{\mathrm{SL}}=-\tau_{P} /\left\langle\log \left(1-\sigma \lambda_{2}\right)\right\rangle
$$

is a reasonable upper bound for the characteristic time, since it can be improved only by the motion of the agents. Figure 2(a) shows that $T_{\mathrm{SL}}$ is not only an upper bound, but also a good approximation for small values of $\tau_{P}$. When $d$ is large enough for the system to form a complete graph, FSA fits again the numerical result.
In real mobile networks, the signal interval $\tau_{P}$ is one of the easiest parameters to control. Thus, it is important to study the dependence of $T$ on $\tau_{P}$ with other parameters fixed. Figure 2(c) describes this dependence. Decreasing $\tau_{P}$ saturates $T$, while increasing $\tau_{P}$ saturates $n_{T}$. Therefore, there exists an optimal value of $\tau_{P}$ in the intermediate region that simultaneously achieves rapid synchronization and high efficiency. This result demonstrates the importance of taking into account the deviation from FSA, since it causes the saturation of $T$.

In order to get some analytical insight, we introduce the normal modes of the linear dynamics (3). Note that $L_{i j}$ changes with time. Let $\theta_{l}(t)$ be the normal modes corresponding to an eigenvalue $\lambda_{l}$ at time $t$, which satisfies $\varphi_{j}(t)=$ $\sum_{l=1}^{N} U_{j l}(t) \theta_{l}(t)$, where $U_{j l}(t)$ is the orthogonal matrix with a unit determinant. Multiplying the two sides of Eq. (3) by the transpose $U_{l i}^{T}\left(t+\tau_{P}\right)$ from the left, we get

$$
\theta_{l}\left(t+\tau_{P}\right)=\sum_{m=1}^{N} O_{l m}(t)\left[1-\sigma \lambda_{m}(t)\right] \theta_{m}(t) .
$$

Here $O_{l m}(t) \equiv \sum_{i} U_{l i}^{T}\left(t+\tau_{P}\right) U_{i m}(t)$ is orthogonal. Then after an arbitrary number of time steps we get

$$
\theta_{l_{n}}\left(t+n \tau_{P}\right)=\prod_{q=0}^{n-1}\left[\sum_{l_{q}=1}^{N} O_{l_{q+1} l_{q}}\left(1-\sigma \lambda_{l_{q}}\right)\right] \theta_{l_{0}}(t),
$$

where $l_{q}$ denotes the suffix corresponding to an eigenmode at time $t+q \tau_{P}$. The product of these matrices separately describes the transformation of the normal modes of instantaneous networks by $O_{l_{q+1} l_{q}}$ and the decay of each eigenmode by $\left(1-\sigma \lambda_{l_{q}}\right)$. 
This product of $n$ matrices in Eq. (8) can be diagonalized. Let its eigenvalues be $\left(1-\sigma \Lambda_{i}\right)^{n}$ with $0=\Lambda_{1} \leqslant \Lambda_{2} \leqslant \cdots \leqslant$ $\Lambda_{N}$; the limit of $\Lambda_{i}$ exists for $n \rightarrow \infty$. The characteristic time can then be written as $T=-\tau_{P} / \log \left(1-\sigma \Lambda_{2}\right)$. Figure 2(c) compares $T$ obtained by this method to that directly measured from simulations. Their agreement is excellent, even for smaller values of $\tau_{P}$ where FSA does not hold. Our procedure can be generalized to any other evolving network [23].

The two nontrivial behaviors obtained in the simulations can also be distinguished in the matrix product formulation. For $\eta \ll 1$, where FSA holds, the agents move a sufficiently long distance during $\tau_{P}$, and $L_{i j}(t)$ and $L_{i j}\left(t+\tau_{P}\right)$ are regarded as independent. Thus, their eigenvalues $\lambda_{l}(t)$ and $\lambda_{l}\left(t+\tau_{P}\right)$ are uncorrelated. Then we can expect $\prod_{q=1}^{n}(1-$ $\left.\sigma \lambda_{l_{q}}\right) \approx e^{n\langle\log (1-\sigma \lambda)\rangle}$ if we neglect the fluctuation, where the bracket represents the average over eigenvalues of the Laplacian matrix. Then we get $T=-\tau_{P} /\langle\log (1-\sigma \lambda)\rangle$. Since the average eigenvalue of the Laplacian matrix is the average degree, we get $\langle\lambda\rangle=(N-1) \rho$. Expanding the characteristic time in powers of $\sigma$, we have

$$
\tau_{P} / T=\sigma(N-1) \rho+\mathcal{O}\left(\sigma^{2}\right),
$$

which is equal to $\tau_{P} / T_{\mathrm{FS}}$ up to the lowest order in $\sigma$.

For $\eta \gg 1$ and $d \ll d_{c}$ (multiple clusters), there is more than one zero eigenmode of the instantaneous Laplacian matrix, and $\theta_{l}(t)$ corresponding to a nonzero eigenvalue $\lambda_{l}(t) \neq 0$ vanishes before the topology changes, implying that local synchronization is achieved. Hence, the dynamics of the system is governed by the decay of the zero eigenmodes caused by the topological change. Even if we increase the number of signals by decreasing $\tau_{P}$, we cannot get a further decrease of the synchronization error between disconnected clusters. Therefore, we expect that $T$ converges to a finite value for $\tau_{P} \rightarrow 0$. Since $\sigma$ appears only in the nonzero eigenmodes $\left(1-\sigma \lambda_{l}\right)$, which are neglected in our approximation, the converged value of $T$ does not depend on $\sigma$ either [Fig. 2(c)].

In summary, we have presented a model of interactions between moving agents that takes into account two different timescales: one related to local synchronization in clusters and the other related to the topology change. We have shown that when the second timescale is greater than the first, more time is required for the system to achieve synchronization than the prediction of FSA. This new effect is particularly important, because it affects the optimal parameter values in terms of synchronization time and efficiency. Although our model assumes purely random motion, it could be easily extended to more realistic patterns of motion [24]. Our result suggests that the interplay between instantaneous topology, agent motion, and interaction rules plays an important role for the performance of mobile systems such as ad hoc networks or sensor networks.

$\mathrm{NF}$ and JK are grateful for financial support from WGL (project ECONS) and FET Open project SUMO (grant agreement 266722). AD-G acknowledges financial support from Spanish MCINN (grants FIS2006-13321, FIS2009-13730, PR2008-0114) and Generalitat de Catalunya (grant 2009SGR00838).
[1] R. Albert and A.-L. Barabási, Rev. Mod. Phys. 74, 47 (2002).

[2] S. Boccaletti, V. Latora, Y. Moreno, M. Chavez, and D.-U. Hwang, Phys. Rep. 424, 175 (2006).

[3] A. Arenas, A. Díaz-Guilera, J. Kurths, Y. Moreno, and C. Zhou, Phys. Rep. 469, 93 (2008).

[4] M. L. Sachtjen, B. A. Carreras, and V. E. Lynch, Phys. Rev. E 61, 4877 (2000).

[5] R. Olfati-Saber, J. A. Fax, and R. M. Murray, Proc. IEEE 95, 215 (2007).

[6] J.-P. Onnela, J. Saramaki, J. Hyvonen, G. Szabo, D. Lazer, K. Kaski, J. Kertesz, and A. L. Barabasi, Proc. Natl. Acad. Sci. USA 104, 7332 (2007).

[7] M. Valencia, J. Martinerie, S. Dupont, and M. Chavez, Phys. Rev. E 77, 050905(R) (2008).

[8] A. Buscarino, L. Fortuna, M. Frasca, and A. Rizzo, Chaos 16, 015116 (2006).

[9] H. G. Tanner, A. Jadbabaie, and G. J. Pappas, in Proceedings, 42nd IEEE Conference on Decision and Control, pp. 2016-2021 (2003).

[10] J. Buhl, D. J. T. Sumpter, I. D. Couzin, J. J. Hale, E. Despland, E. R. Miller, and S. J. Simpson, Science 312, 1402 (2006).

[11] D. Tanaka, Phys. Rev. Lett. 99, 134103 (2007).

[12] K. Römer, in Proceedings of the 2nd ACM International Symposium on Mobile ad hoc Networking \& Computing (ACM, NY, USA, 2001), pp. 173-182.

[13] F. Sivrikaya, and B. Yener, IEEE Network 18, 45 (2004).

[14] I. V. Belykh, V. N. Belykh, and M. Hasler, Physica D 195, 188 (2004).
[15] M. Frasca, A. Buscarino, A. Rizzo, L. Fortuna, and S. Boccaletti, Phys. Rev. Lett. 100, 044102 (2008).

[16] M. Porfiri, D. J. Stilwell, E. M. Bollt, and J. D. Skufca, Physica D 224, 102 (2006).

[17] D. J. Stilwell, E. M. Bollt, and D. G. Roberson, SIAM J. App. Dyn. Syst. 5, 140 (2006).

[18] F. Peruani, E. M. Nicola, and L. G. Morelli, New J. Phys. 12, 093029 (2010).

[19] Y. Kuramoto, Chemical Oscillations, Waves, and Turbulence (Springer, New York, 1984); J. A. Acebrón, L. L. Bonilla, C. J. Pérez-Vicente, F. Ritort, and R. Spigler, Rev. Mod. Phys. 77, 137 (2005).

[20] G. Filatrella, A. H. Nielsen, and N. F. Pedersen, Eur. Phys. J. B 61, 485 (2008); S. Lämmer, H. Kori, K. Peters, and D. Helbing, Physica A 363, 39 (2006); D. J. Klein, P. Lee, K. A. Morgansen, and T. Javidi, IEEE J. Selected Areas Commun. 26, 695 (2008).

[21] J. Dall and M. Christensen, Phys. Rev. E 66, 016121 (2002); P. Balister, B. Bollobás, and M. Walters, Random Struct. Algor. 26, 392 (2005).

[22] See supplemental material at [http://link.aps.org/supplemental/ 10.1103/PhysRevE.83.025101] for movies of the phase updates corresponding to Fig. 1.

[23] S. Boccaletti, D.-U. Hwang, M. Chávez, A. Amann, J. Kurths, and L. M. Pecora, Phys. Rev. E 74, 016102 (2006); J. Zhao, D. J. Hill, and T. Liu, Automatica 45, 2502 (2009).

[24] M. C. Gonzalez, C. A. Hidalgo, and A.-L. Barabasi, Nature (London) 453, 779 (2008). 